\title{
Servicio de mapoteca virtual de la Escuela de Ciencias Geográficas, Universidad Nacional
}

\author{
Virtual map library service of the School of Geographical \\ Sciences, National University
}

\author{
Ricardo Orozco-Montoya ${ }^{1}$ \\ Bepsy Cedeño-Montoya ${ }^{2}$ \\ Universidad Nacional, Costa Rica
}

\begin{abstract}
Resumen
Desde el 2007, la Escuela de Ciencias Geográficas (ECG) de la Universidad Nacional de Costa Rica (UNA) ha apoyado una actividad académica denominada "Servicio de mapoteca virtual para la incorporación de las TIC en las actividades académicas". Sin embargo, el rápido avance de las herramientas para la publicación de información geográfica en la Web, la consolidación de la Infraestructura de Datos Espaciales de Costa Rica (IDECORI) y del Sistema Nacional de Información Territorial (SNIT) como portal de datos geográficos oficiales, últimos dos puntos liderados por el Instituto Geográfico Nacional (IGN) del Registro Nacional de Costa Rica, ha obligado a que se reoriente el quehacer de la Mapoteca Virtual.. En este trabajo se presenta la nueva propuesta que guiará el desarrollo de la plataforma de Mapoteca Virtual, con lo que se busca ofrecer acceso a datos geográficos generados en la UNA, iniciando con la Facultad de Ciencias de la Tierra y el Mar (FCTM), y otros disponibles mediante alianzas estratégicas (como el convenio suscrito con la Dirección General del Archivo Nacional para la publicación de mapas históricos), aplicando ahora los estándares definidos por IDECORI.
\end{abstract}

Palabras clave: Mapoteca Virtual, IDE, Servicios OGC, Cartografía, Cartoteca histórica.

1 Máster, Académico/Investigador Escuela de Ciencias Geográficas, Universidad Nacional, Costa Rica. Correo electrónico: ricardo.orozco.montoya@una.cr

2 Máster, Académico/Investigador Escuela de Ciencias Geográficas, Universidad Nacional, Costa Rica. Correo electrónico: bepsy.cedeno.montoya@una.cr

Este artículo corresponde a la ponencia presentada en el 35th Conference of Latin American Geographers realizada en San José, Costa Rica del 20 al 22 de mayo del 2018 


\begin{abstract}
Since 2007, the School of Geographical Sciences (ECG) of the National University of Costa Rica (UNA) has supported an academic activity called "Virtual map library service for the incorporation of ICT in academic activities". However, the rapid advance of the tools for the publication of geographic information on the Web, the consolidation of the Costa Rican Spatial Data Infrastructure (IDECORI) and the National Territorial Information System (SNIT) as an official geographic data portal, last two points led by the National Geographic Institute (IGN) of the National Registry of Costa Rica, has forced to reorient the work of the Virtual Map Library. This paper presents the new proposal that will guide the development of the Virtual map library platform, with the aim of offering access to geographic data generated in the UNA, starting with the Faculty of Earth and Sea Sciences (FCTM), and others available through strategic alliances (such as the agreement signed with the General Directorate of the National Archive for the publication of historical maps), now applying the standards defined by IDECORI.
\end{abstract}

Keywords: Virtual Map Library, IDE, OGC Services, Cartography, Historical Map Library.

\title{
Introducción
}

La cartografía está emigrando hacia una era digital cada más fuerte, donde los mapas en papel han sido desplazados por mapas de tipo digital que modernizan la representación de los espacios geográficos, es de esta forma que las ciencias de la información geográfica han tomado relevancia en los últimos años con la incorporación de los Sistemas de Información Geográfica (SIG) a la cotidianidad no solo académica, si no institucional y estatal en general.

Con el desarrollo de las Tecnologías de Información y Comunicación (TIC) se volvió imprescindible que las actividades académicas incorporen dichas tecnologías en sus quehaceres, además el desarrollo de los SIG mediante la revolución intelectual del espacio geográfico ha perfilado lo que podría denominarse una nueva geografía aplicada, que incluye campos emergentes como la geografía automatizada, la cibergeografía, la geoinformática y la geografía global, las cuales tienen como punto común la estandarización de datos espaciales de forma digital, para difundirse mediante internet en diversas prácticas disciplinarias (Buzai, 2012).

Es así como el desarrollo creciente de las tecnologías asociadas a la información espacial empuja a distintos profesionales al desarrollo de capacidades en el uso de las denominadas Tecnologías de Información Geográfica (TIG), entre las que se destacan: imágenes satelitales, mapas digitales y uso de sistemas de posicionamiento global (GPS), los cuales se han convertido en una oportunidad para modernizar las actividades 
académicas (Barrantes, 2011). El uso de este tipo de información permite el desarrollo y la formación del pensamiento espacial y el pensamiento geográfico en distintos profesionales, donde el primero es ligado más que todo a las características físicas de ese espacio, mientras que el segundo hace una relación de ese espacio físico con su dimensión humana, social, económica y política (González, 2015).

Es de esta forma como la transformación del mundo real en un mundo digital visto en monitores y manipulado mediante procedimientos computacionales (Buzai, 2001), ha llevado a la búsqueda de servicios digitales que permitan incorporar las TIC y las TIG en las actividades académicas para el desarrollo de competencias espaciales y situacionales del espacio geográfico, con la finalidad de que dicho espacio sea estudiado desde su integralidad biofísica y socioeconómica.

Dada la necesidad de consulta de información histórica y actualizada de un espacio geográfico determinado y la dispersión de información tanto en formato analógico como digital, es que surgen los nuevos requerimientos para el almacenamiento de datos cartográficos, por lo cual se han creado las mapotecas o cartotecas digitales que permiten el almacenamiento, búsqueda y recuperación de mapas digitales (Barrantes et al., 2010).

Desde hace varios años las mapotecas o cartotecas dentro de museos, universidades y bibliotecas se han convertido en algo común, principalmente en países desarrollados con cartografía histórica importante que han logrado convertir a formato digital. Algunos países latinoamericanos como Chile, Argentina, México y Colombia que posee una larga tradición cartográfica, han iniciado la migración hacia mapotecas virtuales (Quesada y Barrantes, 2016).

Es así como desde el 2007, la Escuela de Ciencias Geográficas de la Universidad Nacional en Costa Rica, desarrolló el proyecto denominado "Servicio de mapoteca virtual para el apoyo de la docencia y la investigación", el cual se ha convertido en una actividad académica permanente que desde el 2015 es denominado "Servicio de mapoteca virtual para la incorporación de las TIC en las actividades académicas". Este servicio se ofrece desde una plataforma digital parte del sitio web de la Universidad Nacional (UNA) y pretende por medio de su formulación como actividad permanente, dar mantenimiento y desarrollo al sitio web de la mapoteca virtual para la ampliación de servicios y colecciones de mapas digitales 
que le brinde a la docencia e investigación en la UNA, una herramienta para incluir las TIC y TIG en su quehacer académico.

La mapoteca virtual de la Escuela de Ciencias Geográficas de la UNA difunde el uso del formato digital entre sus usuarios y procura crear nueva información cartográfica que retroalimente la colección. $\mathrm{N}$ obstante, tal como indica Barrantes et al., (2010), el crecimiento y mantenimiento futuro de dicha colección, dependerá de los aportes de los autores cartográficas, desde distintas áreas como geografía, geología, ecología, hidrología, demografía, economía, entre otras, que contribuyen a agrandar la colección mediante el acceso abierto a publicaciones científicas.

El rápido avance de las herramientas para la publicación de información geográfica en la Web, la consolidación de la Infraestructura de Datos Espaciales de Costa Rica (IDECORI) y del Sistema Nacional de Información Territorial (SNIT) como portal de datos geográficos oficiales, últimos dos puntos liderados por el Instituto Geográfico Nacional (IGN) del Registro Nacional de Costa Rica, ha obligado a que se reoriente el quehacer de la Mapoteca Virtual, por lo cual, el presente trabajo presenta la nueva propuesta que guiará el desarrollo de la plataforma, con el objetivo de desarrollar un servicio de mapoteca virtual que ponga a disposición del público en general, los datos geográficos generados en la UNA y otras instituciones nacionales o internacionales generadoras de datos espaciales.

\section{Reorientación de la Mapoteca Virtual}

Desde sus inicios, el servicio de mapoteca virtual ha puesto ha disposición del público en general una amplia publicación de mapas digitales producidos por distintas institucionales gubernamentales y productos de trabajos científicos desarrollados en la UNA, además sus servicios han aumentado con la publicación de manuales sobre técnicas cartográficas y el uso de los SIG, y más recientemente la generación de productos cartográficos a través del software libre relacionado con los SIG (Quesada, 2015).

La mapoteca virtual de la Escuela de Ciencias Geográficas, ha sido caracterizada por una colección importante de datos espaciales, tanto en formato de capas de información (vectoriales o raster) como mapas en digital, la cual inició con productos provenientes de proyectos científicos universitarios, investigaciones académicas y trabajos de laboratorios de docencia, los cuales se han complementado con la información proveniente 
de instituciones y organizaciones no gubernamentales que colaboraron con la inclusión de cartografía (Barrantes, 2011). Sin embargo, el desarrollo de nuevas herramientas para la obtención de datos geográficos y las TIG en general, además de la implementación de la IDECORI por parte del IGN como sitio oficial de la cartografía nacional, ha provocado que el servicio de mapoteca virtual reoriente su quehacer para cumplir con su objetivo y la legislación nacional.

El establecimiento en Costa Rica de una Infraestructura de Datos Espaciales (IDE) de manera oficial, obliga a la mapoteca virtual de la Escuela de Ciencias Geográficas a cumplir con dichos estándares y normativas, ya que de acuerdo con Olaya (2014), una IDE puede definirse como un conjunto de datos espaciales, tecnología, normas y planes institucionales encaminados a facilitar la disponibilidad y el acceso a dichos datos. De esta forma la mapoteca virtual podría convertirse en un componente importante de la IDECORI.

Una IDE permite hacer que exista una información geográfica armonizada y de alta calidad, asequible y disponible a usuarios para formular, implementar, controlar y evaluar políticas y normativas relacionadas al tema espacial (Pascual, 2015). Para que lo anterior pueda darse, es importante que funcionen de manera articulada tres niveles de una IDE según Hernández-López et al., (2013): el primer nivel consiste en los datos espaciales ordenados en diferentes formatos, el segundo, se refiere a los servidores de aplicaciones o servicios web de interfaz que permiten el acceso de la información, y el tercer nivel es el usuario final que hace uso de los datos espaciales. Precisamente los niveles o mecanismos de una IDE propuestos por Olaya (2014) se resumen en el cuadro 1. 
Cuadro 1: Mecanismos de una IDE

\begin{tabular}{|l|l|}
\hline \multicolumn{1}{|c|}{ Mecanismo } & \multicolumn{1}{c|}{ Descripción } \\
\hline Información contenida & $\begin{array}{l}\text {-Conjunto de datos espaciales } \\
\text {-Servicio de catálogo } \\
\text {-Servicios de acceso a la información (Servicios Open } \\
\text { Geospatial Consortium-OGC) } \\
\text {-Metadatos }\end{array}$ \\
\hline Utilización de Estándares & $\begin{array}{l}\text {-Estándares aceptados internacionalmente para la } \\
\text { interoperabilidad técnica de la IDE }\end{array}$ \\
\hline Establecimiento de acuerdos & $\begin{array}{l}\text {-Acuerdos entre actores para la utilización de } \\
\text { tecnologías y servicios compatibles y unificación de } \\
\text { modelos de datos. }\end{array}$ \\
\hline
\end{tabular}

Fuente: Olaya (2014).

En Costa Rica, a partir del 2013 se crea el Sistema Nacional de Información Territorial (SNIT), el cual ha sido un punto clave en el proceso de consolidación de la IDECORI, la cual es definida como:

“...el conjunto de políticas, organizaciones, estándares y tecnologías que trabajan en forma conjunta para producir, compartir y usar la información geográfica necesaria para la planificación y el ordenamiento territorial, la seguridad nacional y el desarrollo del país en diversos ámbitos.” (SNIT, 2018).

Es basado en lo anterior que se designa a la IDECORI y al SNIT como la plataforma oficial para la publicación de información geográfica y la que contiene todos los estándares y normas para la generación de datos geográficos en el ámbito nacional, regional y local, con la finalidad de que la información sea accesible rápidamente para todas aquellas entidades que son usuarias y generadoras de información geográfica (SNIT, 2018). Además de acuerdo con el decreto $\mathrm{N}^{\circ}$ 37773-JP-H-MINAE-MICITT, publicado en La Gaceta N ${ }^{\circ} 134$ del 12 de julio del 2013, se indica que el SNIT se convierte en punto central de acceso a datos abiertos en el que se enmarca la institucionalidad del país que genera información geográfica, cumpliendo así una función primordial dentro los procesos de transparencia y rendición de cuentas.

Dada la información anterior, la Mapoteca Virtual en su afán de ser una herramienta eficaz que reúna información geográfica estandarizada y 
apegada a la oficialidad del país, que sea de acceso libre a usuarios y apoye las labores de docencia, investigación y extensión de la UNA, es que la misma se encuentra en un proceso de reorientación para complementar a la IDECORI y brindar un servicio novedoso y útil a los distintos usuarios que no sea una duplicación del SNIT y más bien se convierta en un componente más del mismo.

Es de esta forma como el desarrollo de la nueva plataforma de Mapoteca Virtual busca ofrecer acceso a datos geográficos generados en la UNA mediante investigaciones académicas, iniciando con la Facultad de Ciencias de la Tierra y el Mar (FCTM), y otros disponibles mediante alianzas estratégicas (como el convenio suscrito con la Dirección General del Archivo Nacional para la publicación de mapas históricos), aplicando ahora los estándares definidos por IDECORI.

\section{Propuesta para el desarrollo de la plataforma de la nueva Ma- poteca Virtual}

El desarrollo de la plataforma de la nueva mapoteca virtual se basará en su reorientación dirigida hacia el almacenamiento de los datos geográficos generados en la UNA, iniciando con la FCTM y de ahí de forma sucesiva con otras facultades e institutos de investigación, siendo de esta manera un centro de información que cumpla con los estándares de la IDECORI y convertirse a futuro en uno de sus componentes principales de información, de tal forma que la UNA sea un nodo más del SNIT y cuente con un espacio en el sitio oficial de almacenaje y descarga de información.

Para la generación de la plataforma de la nueva mapoteca virtual se han planteado tres objetivos específicos que serán la guía para el desarrollo de esta:

1. Ofrecer acceso a datos geográficos generados en la UNA y otras instituciones nacionales o internacionales productoras de datos espaciales, mediante el servicio de mapoteca virtual.

2. Fomentar el uso de las TIC y las TIG en los procesos de docencia, investigación y extensión de la Facultad de Ciencias de la Tierra y el Mar.

3. Divulgar el servicio ofrecido a través de la mapoteca virtual, para su uso tanto a nivel educativo, profesional y al público en general. 
Basado en los objetivos anteriores, se han planteado cinco grandes actividades que se resumen en el cuadro 2 .

Cuadro 2: Propuesta de rediseño de nueva Mapoteca Virtual

\begin{tabular}{|c|c|}
\hline Actividades & Tareas \\
\hline $\begin{array}{l}\text { Página Web de Mapoteca } \\
\text { Virtual }\end{array}$ & $\begin{array}{l}\text { - Rediseñar y publicar la página web de Mapoteca Virtual } \\
\text { de la Escuela de Ciencias Geográficas. }\end{array}$ \\
\hline $\begin{array}{l}\text { Repositorio de datos } \\
\text { espaciales de la Escuela de } \\
\text { Ciencias Geográficas }\end{array}$ & $\begin{array}{l}\text { - Creación de un repositorio de datos espaciales para la } \\
\text { Escuela de Ciencias Geográficas. } \\
\text { - Establecer acciones de cooperación con el proyecto } \\
\text { UNA RED-IDE FCTM, con el fin de seguir apoyando } \\
\text { la estandarización de datos geográficos generados en la } \\
\text { FCTM. }\end{array}$ \\
\hline Cartoteca institucional & $\begin{array}{l}\text { - Proveer acceso a la colección de mapas históricos } \\
\text { escaneados del Archivo Nacional, mediante el servicio } \\
\text { de cartoteca histórica. }\end{array}$ \\
\hline Divulgación & $\begin{array}{l}\text { - Ofrecer capacitación en el acceso y uso de datos } \\
\text { geográficos en línea. } \\
\text { - Divulgar el quehacer y servicios de la Mapoteca Virtual. }\end{array}$ \\
\hline Visor de datos geográficos & $\begin{array}{l}\text { - Publicar en la web un visor de datos geográficos para } \\
\text { los mapas históricos georreferenciados. }\end{array}$ \\
\hline
\end{tabular}

El rediseño y publicación de la nueva página web de la mapoteca virtual tendrá la misma concepción que sus inicios, dirigida hacia el acceso abierto a publicaciones científicas, en este caso cartografía digital, con el propósito de beneficiar a la sociedad y sobre todo estudiantes y docentes universitarios, con la posibilidad de compartir mapas digitales (Barrantes, 2011) y acceder a información de proyectos de la UNA.

En el caso del repositorio de datos espaciales de la Escuela de Ciencias Geográficas (ECG), se busca contar con una colección de datos espaciales generados o adquiridos por los proyectos académicos que se desarrollan en la ECG, tanto en forma de capas de información como mapas digitales. Esta iniciativa podrá hacerse extensiva a otras unidades académicas de la FCTM, de forma tal que se cuente con un punto central de acceso a datos geográficos generados por la facultad, difundiendo de esta forma el quehacer académico y su fácil acceso.

La cartoteca institucional es una de las actividades más importantes de la mapoteca virtual, ya que a partir del 2013 se firmó un convenio 
específico entre la UNA y la Dirección General del Archivo Nacional (DGAN) para facilitación de un escáner de alta resolución por parte de la ECG para escanear lo mapas y planos antiguos, con la finalidad de ponerlos a disposición del público en general a través de la página web de la mapoteca virtual, de esta forma se ha llegado al desarrollo de una cartoteca histórica de Costa Rica en un marco de colaboración entre dos instituciones (Quesada y Barrantes, 2016).

El servicio de mapoteca virtual de la ECG busca estrategias de divulgación entre sus usuarios, principalmente a lo interno de la UNA y actividades académicas y científicas externas como simposios, encuentros y congresos, donde la comunidad científica nacional e internacional se informan acerca de esta iniciativa y sus beneficios en cuanto al acceso de información.

Finalmente, el servicio de mapoteca virtual apunta hacia el diseño y puesta en marcha de un visor geográfico en línea de distintas capas de información de los proyectos desarrollados en la FCTM, adaptados estos a los requerimientos de la IDECORI y cumpliendo estándares internacionales de intercambio de información definidos por medio de los servicios Open Geospatial Consortium (OGC), donde los más utilizados son los Servicios de Web de Mapas (WMS), los Servicios Web de Características (WFS) y los Servicios Web de Coberturas (WCS) (Campos-Vargas et al., 2015).

Dadas las anteriores actividades y tareas para el desarrollo de la plataforma de la nueva mapoteca virtual, se busca que por medio del rediseño y publicación de la nueva página web, se logre articular cada una de las demás actividades y que el sitio web se convierta en la representación tangible del servicio de mapoteca virtual, el cual pretende ser una herramienta eficaz para el desarrollo de la docencia, investigación y extensión de la UNA, así como un servicio de fácil acceso de información para la sociedad en general.

\section{Conclusiones}

El servicio de mapoteca virtual de la ECG debe cumplir con estándares nacionales e internacionales, por lo cual su rediseño y reorientación es de vital importancia para apegarse a las normativas y ser un sitio de interés para investigadores científicos, estudiantes, profesionales institucionales y sociedad en general. Es de esta forma como la importancia del servicio se dirige hacia la concentración de información de proyectos con datos espaciales desarrollados en la UNA, y que logren difundirse a través de la 
página web de la mapoteca virtual con la finalidad de divulgar el quehacer académico y su utilidad para esfuerzos del país en la transparencia, rendición de cuentas y acceso a la información generada con fondos públicos.

A futuro este servicio podría convertirse en un actor o nodo importante del SNIT, siendo parte del portal web oficial del país en temas de datos espaciales, ya que actualmente sigue los estándares de la IDECORI. Además, la mapoteca virtual podría convertirse en el visor en línea oficial de la UNA para la visualización de datos geográficos de los proyectos de investigación, extensión y docencia que se desarrollen en el país por medio de fondos de la UNA.

Definitivamente el servicio de mapoteca virtual es una herramienta de apoyo en las labores docentes y académicas en general, ya que por medio del uso de las TIC y las TIG en su concepción, apoya la acción sustantiva de la UNA correspondiente a lo académico, donde a través de los mapas digitales y productos finales de investigaciones científicas que contengan información geográfica, se actúa en el desarrollo y la formación del pensamiento espacial y el pensamiento geográfico en estudiantes de distintas disciplinas.

Finalmente, el desarrollo de la cartoteca institucional es una herramienta de gran interés histórico y cultural, ya que la mapoteca virtual se convertiría en el único sitio web del país que contendrá una colección tan grande de mapas históricos, lo cual apoyará las investigaciones históricas y resolución de conflictos en temas territoriales.

\section{Referencias}

Barrantes, G.; Tercero, J.; Picado, A.; \& Morera, C. (2010). La Mapoteca

Virtual: un ejemplo de colaboración bajo una plataforma de software libre. Revista geográfica de América Central, (45): 65-76.

Barrantes, G. (2011) Mapoteca Virtual: una herramienta tecnológica en apoyo a la educación. Revista geográfica de América Central, número especial EGAL 2011: 1-10.

Buzai, G. (2001). Perspectivas digitales: visiones espaciales y carta a los futuros usuarios de sistemas de información geográfica. Huellas, (6): 11-37. Buzai, G. (2012). Geografía y sistemas de información geografía: evolución teórico-metodológica hacia campos emergentes. Revista geográfica de América Central, número especial: 15-67. 
Campos-Vargas, C.; Mora-Zamora, R.; \& Segura-Castillo, A. (2015). Geovisión: una infraestructura abierta de datos espaciales. Tecnología en marcha, 28 (3): 15-24.

González, R. (2015). Del pensamiento espacial al conocimiento geográfico a través del aprendizaje activo con tecnologías de la información geográfica. Giramundo, 2 (4): 7-13.

Hernández-López, D.; Felipe-García, B.; González-Aguilera, D.; \& AriasPérez, B. (2013). Web-based spatial data infrastructure: a solution for the sustainable management of thematic Information supported by aerial orthophotography. Dyna, 80 (178): 123-131.

Olaya, V. (2014). Sistemas de Información Geográfica. Creative Common Atribución. Recuperado de: https://www.icog.es/TyT/files/Libro_ SIG.pdf.

Pascual, N. (2015). Posibilidades didácticas de las infraestructuras de datos espaciales: Propuesta de aplicación didáctica de IDERioja. Contextos Educativos, (18): 109-128.

Quesada, A. (2015). La Mapoteca Virtual de la Universidad Nacional de Costa Rica. Revista Electrónica Perspectivas, (11): 1-13.

Quesada, A. \& Barrantes, G. (2016). La cartoteca histórica de Costa Rica, un medio para la protección y divulgación del patrimonio cartográfico. Diálogos, 17 (1): 143-155.

Sistema Nacional de Información Territorial (SNIT). (2018). Infraestructura Nacional de Datos Espaciales de Costa Rica. Recuperado de: www.snitcr.go.cr 
